Enfoques. Revista de Investigación en Ciencias de la Administración http://doi.org/10.33996/revistaenfoques.v5i18.112

\title{
PLAN DE NEGOCIOS PARA LA COOPERATIVA INDUSTRIAL DE CALZADO PARA FORTALECER SU IMAGEN CORPORATIVA
}

\author{
BUSINESS PLAN FOR THE FOOTWEAR INDUSTRIAL COOPERATIVE TO \\ STRENGTHEN ITS CORPORATE IMAGE
}

\author{
PLANO DE NEGÓCIOS DA COOPERATIVA INDUSTRIAL CALÇADISTA PARA \\ FORTALECER SUA IMAGEM CORPORATIVA
}

\author{
Cecilia Cristina Mendoza Bazantes \\ Elsa Elisa Estrada Miño Colcha \\ Oscar Paul Tanqueño Colcha
}

Artículo recibido 20 de enero 2021 | Arbitrado 22 de febrero 2021 | Publicado 01 de abril 2021

\section{Resumen}

La investigación tuvo como objetivo principal diseñar, elaborar e implementar un plan de negocios en la cooperativa industrial de Calzado Penipe, para fortalecer su imagen corporativa en la ciudad de Riobamba; con una metodología de tipo Proyecto factible, bajo un enfoque cualitativo y cuantitativo, el diseño que se usó fue cuasi experimental, y el enfoque deductivo e inductivo, la técnica e instrumentos utilizados para la recolección de datos, fueron la observación y la encuesta tanto para el cliente como para los empleados de la empresa. La población y la muestra con la que se trabajo fue de 118.982 habitantes de la ciudad de Riobamba, solo se usaron de muestra 96 personas. Y 10 personas que laboran en la empresa. Dando como resultado, la necesidad de la aplicación de un plan de negocios para ayudar a mejorar la empresa. Así que, se logró aumentar las ventas, entre otros aspecto.

Palabras clave: Estructura de negocios; imagen corporativa; plan de negocios; plan de marketing, plan financiero

\begin{abstract}
The main objective of the investigation was to design, elaborate and implement a business plan in the Calzado Penipe industrial cooperative, to strengthen its corporate image in the city of Riobamba; With a feasible Project type methodology, under a qualitative and quantitative approach, the design used was quasi-experimental, and the deductive and inductive approach, the technique and instruments used for data collection, were the observation and the survey both for the client as well as the company's employees. The population and the sample with which we worked was 118,982 inhabitants of the city of Riobamba, only 96 people were used as a sample. And 10 people who work in the company. Resulting in the need for the implementation of a business plan to help improve the company. So, it was possible to increase sales, among other aspects.
\end{abstract}

Key words: Business structure; corporate image; business plan; marketing plan, financial plan
Cecilia Cristina Mendoza Bazantes

mibonitacriss1986@gmail.com

Orcid: 0000-0003-1276-5847

Universidad Nacional de Chimborazo, Riobamba, Ecuador.

Magíster en Pequeñas y Medianas Empresas Mención Finanzas. Ingeniera en Contabilidad y Auditoría CPA, Ingeniera en Administración de Empresas. Docente de la Universidad Nacional de Chimborazo. Investigadora y tutora de varias tesis de pregrado y posgrado. Universidad Nacional de Chimborazo, Ecuador.

Elsa Elisa Estrada Miño Colcha elyestrada7@gmail.com Orcid: 0000-0002-3503-4003

Universidad Estatal de Bolívar, Bolívar, Ecuador.

Ingeniera en Comercio Exterior Mención en Negociaciones Internacionales, en la Escuela Superior Politécnica de Chimborazo, Magister en Marketing y Servicio al Cliente, Experiencia laboral en la Administración estatal Pública En el área financiera del Ban Ecuador, Docente Investigadora y miembro del tribunal de varias tesis de pregrado, de la Universidad Estatal de Bolívar y Conferencista en, empresas públicas y privadas del Ecuador y Perú. Universidad Estatal de Bolívar, Ecuador.

Oscar Paul Tanqueño Colcha oscarpaul1982@hotmail.com Orcid: 0000-0002-5718-335X

Universidad Estatal de Bolívar, Bolívar, Ecuador.

Ingeniero de empresas. Asistente de cátedra con méritos de beca académica aprobada por la dirección de bienestar estudiantil de la Escuela Superior Politécnica de Chimborazo. Magister en Contabilidad y Auditoría en la Universidad Nacional de Chimborazo concatenado a un proyecto de vinculación. Subgerente de microcrédito en Banco Solidario por cinco años. Actualmente docente en la Facultad de Ciencias Administrativas de la Universidad Estatal de Bolivar, con gestión en proyectos de investigación y diseño de nueva oferta académica. Universidad Estatal de Bolivar, Ecuador. 


\section{Resumo}

O objetivo principal da pesquisa foi desenhar, elaborar e implementar um plano de negócios na cooperativa industrial Calzado Penipe, para fortalecer sua imagem corporativa na cidade de Riobamba; Com uma metodologia do tipo Projeto viável, sob uma abordagem qualitativa e quantitativa, o desenho utilizado foi quase experimental, e a abordagem dedutiva e indutiva, a técnica e os instrumentos utilizados para a coleta de dados, foram a observação e a pesquisa tanto para o cliente como funcionários da empresa. A população e a amostra com a qual trabalhamos foi de 118.982 habitantes da cidade de Riobamba, apenas 96 pessoas foram utilizadas como amostra. E 10 pessoas que trabalham na empresa. Resultando na necessidade da implementação de um plano de negócios para ajudar a melhorar a empresa. Assim, foi possível aumentar as vendas, entre outros aspectos.

Palavras chave: Estrutura empresarial; imagem corporativa; plano de negócios; plano de marketing, plano financeiro

\section{INTRODUCCIÓN}

Partiendo de Vanella (2012), el plan de negocios es considerado como un documento, realizado con el objetivo de describir el fin del negocio y el que los inversionistas se han propuestos, el cual, sigue un proceso secuencial, paulatino, objetivo, relacionado y encaminado a la operación, se detallan además las gestiones futuras que deberán realizar tanto los inversionistas como los clientes internos del negocio considerado los recursos, procurando resultados requeridos.

Además Villarán (2009), indica, que las empresas en marcha van aumentando sus unidades de negocios con la finalidad de crecer y ser más rentables. Sin embargo, un crecimiento no planificado ni controlado podría causar el fracaso de esta nueva unidad de negocio, o lo que es peor, la quiebra de toda la empresa.
De tal manera, un Plan de Negocios tiene algunos estudios dentro de sí mismo como son: plan de operaciones, plan de organización, plan de marketing, y un plan financiero.

En este mismo orden de ideas, la Cooperativa de Producción Industrial del Calzado Penipe "Calzado Vinicio" tiene la responsabilidad de, responder por la calidad de los servicios; de construir una nueva realidad con la que se rompe el dilema : aquel que intenta adaptar al discapacitado /a una realidad de personas sin discapacidades; la alianza de personas en busca de crear una cultura solidaria, el desarrollo económico justo y equitativo de la sociedad y el pleno goce de los derechos humanos y poder producir eficiente y rentablemente, para construir una sociedad con menos pobreza y más solidaria.

Dado a que, la cooperativa no tiene un manejo adecuado y organizado de sus actividades de producción, no posee un manual de funciones actualizado, detallando funciones y deberes de cada uno de los clientes internos, tiene poca publicidad y bajo consumo de sus productos, ocasionando que no se elevan sus ventas porque carece de un fortalecimiento de su imagen corporativa, por tal razón, la investigación pretende diseñar, elaborar e implementar un plan de negocios para alcanzar los objetivos de la cooperativa. Las estrategias permitirán alcanzar dichos objetivos.

Así que, la presente investigación tiene por objetivo principal, el diseño, la elaboración e implementación de un plan de negocios en la cooperativa industrial de Calzado Penipe, para fortalecer su imagen corporativa en la ciudad de Riobamba; está conformado por: un plan organizacional, 
marketing, operacional y financiero, cada uno contiene sus respectivas etapas y la aplicación de diferentes estrategias que ayudan el fortalecimiento de la imagen corporativa e incremento de sus ventas.

La investigación permite fortalecer la imagen de la corporación en la ciudad de Riobamba, además, el producto puede ser conocido por los clientes a través de un adecuado posicionamiento del producto en el mercado, ayuda al desarrollo óptimo de las actividades productivas y de gestión de la cooperativa, aplicando eficiencia, eficacia y efectividad en sus recursos y logrando satisfacción en los clientes.

\section{Marco teórico}

\section{Plan de negocios}

El Plan de Negocios es aquel documento que compendia de forma precisa la información necesaria para determinar si el nuevo negocio es ideal o mejor implementar la actividad de la que produce los rendimientos requeridos y si la misma va a tener el éxito (Corporación Financiera Nacional, 2013).

\section{Estructura del plan de negocios}

Un Plan de Negocios tiene algunos estudios dentro de sí mismo como son:

Plan de operaciones. El empresario puede haber convencido al lector que el producto o servicio ofrecido es superior al de la competencia pero existen los suficientes clientes que estarían dispuestos a comprarlos a un precio justo y valido. Pero los inversionistas saben que si el empresario no cuenta con la infraestructura adecuada, en una ubicación adecuada y si no tiene un sistema eficiente de producción y distribución, es difícil que el negocio sea sostenible y rentable, a pesar de las características del producto o servicio. (Villarán, 2009)

Plan de organización. Dentro de este plan es fundamental establecer los parámetros adecuados para que la empresa se encuentre adecuadamente organizada, es decir que no exista demoras en los procesos, ya que cuenta con un factor humano totalmente capacitado en su área y cumplidor con las normativas legales vigentes a fin de que la empresa pueda cumplir con los objetivos de la misma, con ello tener una carta de presentación para los accionistas y posibles inversionistas. (Ruiz, 2011).

Procesos administrativos. Proceso administrativo es el conjunto de fases o etapas sucesivas a través de las cuales se efectúa la administración de la empresas, las cuales interrelacionan y forman un proceso integral en todas sus áreas. Según el libro administración, una perspectiva global de los autores padres de la administración, las funciones del administrador son: la planificación, organización, dirección y el control que conforman el proceso administrativo cuando se les considera desde el punto de vista sistemático. Fayol señala que estos elementos se aplican en todo tipo de negocios, empresas, políticas, religiosas, filantrópicas y militares. (Monroy, 2014).

Plan de marketing. Un plan de marketing va a permitir a una empresa fortalecer su imagen corporativa, y con ello lograr estrategias adecuadas para poder lograr un incremento paulatino en sus ventas, que permitirá consolidad a la empresa dentro del mercado como una de las mejores. (Almoguera, 2009). Además, es un componente escrito del plan de negocios, que pone en manifiesto la 
situación de cualquier organización con el fin de alcanzar determinada meta, generalmente las estrategias se basan en las "4ps" del marketing (Mullins, Walker, Boyd, y Larreche, 2005).
Estrategias de marketing. Las estrategias de marketing se refieren al conjunto de estrategias denominadas las " $4 p$ ", las cuales se las detalla a continuación:

\section{Estrategias de marketing}

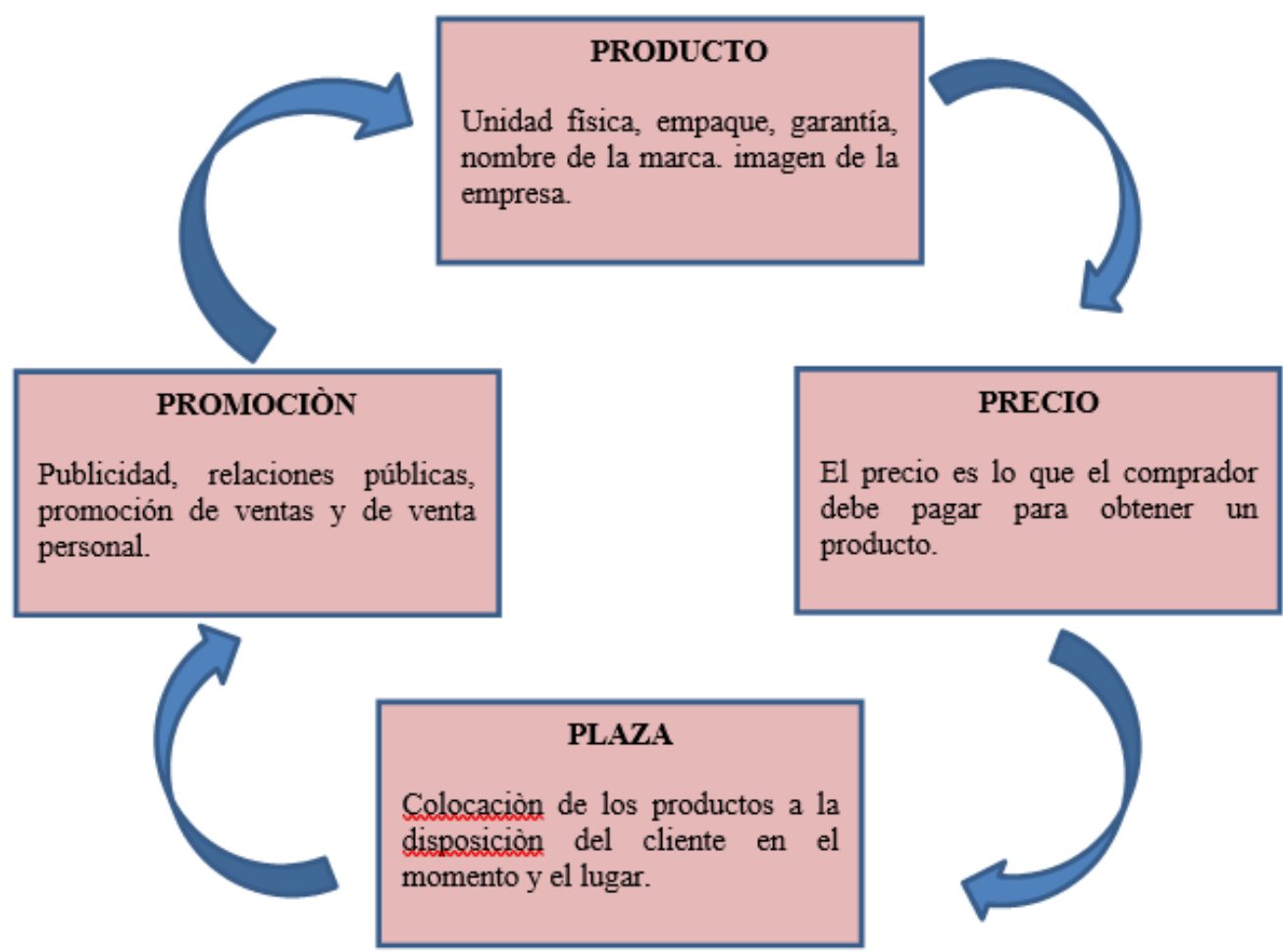

Gráfico 1. Estrategias de marketing. Fuente: (Lamb, hayr, \& Mc. Daniel, 2011).

Objetivos del marketing. Según Villarán (2009), el objetivo del plan de marketing es el de ventas en el que debe establecerse en función a la demanda estimada en el sondeo de mercado, a las ideas del empresario y a la capacidad de producción y endeudamiento de la empresa. Este objetivo debe plantearse en términos monetarios. Otros objetivos fundamentales del plan de marketing: el portafolio de productos, los niveles de la satisfacción de los clientes, el objetivo de participación del producto en el mercado, entre otros.

Estudio de mercado. Mediante el cual se puede obtener información interna que la empresa posee ejemplo la cantidad a producir, precio de venta, con el fin de conseguir orientación para la acertada toma de decisiones del emprendedor. (Martínez, 2013). 
- Mercado. Es el conjunto de clientes que desean el producto o servicio con deseos de satisfacer esas necesidades. (Martínez, 2013).

- Oferta. Cantidad de bienes y/o servicios que los productores están dispuestos a vender en el mercado a cambio de dinero. (Pujol, 2009).

- Demanda. Valor global que expresa la intención de compra de una colectividad. (Pujol, 2009).

- Cliente. El Cliente es la persona más importante de cualquier organización, es la razón de ser. Es el usuario del producto y/o servicio que genera y que se espera satisfacer sus necesidades.

- Marca. Se compone de símbolos, logotipo, anagrama y color que permite identificar a la gama de los productos o servicios de ofrecer un vendedor $\mathrm{o}$ grupo de vendedores y diferenciarlos de los de la competencia. La utilización de marcas presenta ventajas tanto para el consumidor como para el vendedor. Al consumidor le facilita la identificación de los productos y servicios, y le garantiza una calidad comparable cuando repitan la compra. Al vendedor le ayuda a diferenciar sus productos, siendo así más difícil entrar en una guerra de precios. (Pujol, 2009)

Fábrica de calzado. Una fábrica de calzado es una estructura diseñada para la elaboración del producto como parte de la vestimenta de las personas. Posee maquinaria especializada para la construcción de los productos. Consta de las siguientes áreas: cortado, descarnado, costura, pega, rematado, montura y limpieza; que constituyen el proceso, de inicio a fin, de la fabricación de los zapatos; para posteriormente comercializarlo.
Plan financiero. Este plan es la cuantificación en términos monetarios del planteamiento estratégico del negocio. Es el estudio que refleja la estimación del resultado de explotación de la empresa, así como el origen de sus recursos propios y ajenos. Se trata de estudiar "apriori": por un lado, la rentabilidad del aspecto económico del proyecto si genera por sí mismo los recursos necesarios que le permitan desarrollar su actividad comercial en el futuro; por otro, la rentabilidad financiera si la plusvalía que genera la inversión compensa el esfuerzo realizado. (Almoguera, 2009)

Objetivo del plan financiero. El objetivo principal del plan financiero es determinar los recursos que son necesarios para desarrollar la actividad del negocio. El Plan Financiero debe responder 4 interrogantes: $¿ E s$ viable el negocio? ¿Cuánto financiamiento requiero $\mathrm{y}$ cuándo será necesario obtenerlo? ¿Qué tipo de financiamiento se necesita? ¿Quién proveerá el financiamiento? (Almoguera, 2009).

Evaluación financiera. Es aquella que toma en consideración la manera como se obtiene y pagan los recursos financieros utilizados en el proyecto, sin considerar el modo como se distribuyen las utilidades que ha generado el mismo. Los costos, gastos y utilidades constituyen el flujo financiero. (Almoguera, 2009).

Flujo de caja. O llamado también estado de flujo de caja, es el estado financiero que muestra el efectivo generado y utilizado en cada una de las actividades de la operación, inversión y financiamiento. Este estado tiene mayor sentido práctico como presupuesto, siendo una herramienta de vital importancia dentro de la planificación financiera a corto plazo (Sapag, 2007). 
Rentabilidad. Es la relación generalmente expresada en porcentajes, se establece entre el rendimiento económico que proporciona una determinada operación y los que ha invertido en ella. En títulos valores se mide calculando los dineros percibidos (en el caso de las acciones), además de la valorización según su cotización, así como las ventajas a obtenerse por el carácter preferente del incremento del capital (Sapag, 2007).

Evaluación económica. La evaluación económica es un método de análisis útil para adoptar decisiones racionales ante diferentes alternativas. La evaluación económica integra en su análisis tanto los costes monetarios como los beneficios expresados en otras unidades relacionadas con las mejoras en las condiciones de vida de un grupo.

\section{Imagen Corporativa}

La imagen corporativa es lo que la compañía significa, es un ejercicio en la dirección de percepción del espectador, la imagen corporativa es la carta de presentación, para que funcione y ayude a obtener confianza de nuestro público debe tener requisitos básicos: debe reflejar los valores de nuestra empresa, nuestra personalidad debe estar comunicada en nuestra imagen. (Duarte, 2012).

La imagen corporativa está formada por varios componentes que al trabajar conjuntamente que permiten posicionar a la empresa en la mentalidad del cliente. Entre ellos son:

- El nombre de la empresa. Del nombre depende la primera impresión que los clientes se llevan de una empresa, donde se aplica el Plan de negocios es la cooperativa Industrial de "Calzado Penipe" Calzado Vinicio.

- El logo. Puede ser compuesto de palabras, isotipo es de imágenes y el isologotipo que combina tipografía e imagen. El logo debe ser comprensible por el público y atractivo para los potenciales clientes.

- El eslogan. Para que tenga efecto, es necesario que la Cooperativa Industrial Calzado Penipe Calzado Vinicio dé a conocer sobre los beneficios del producto o servicio que su empresa ofrece y que los diferencia de la competencia.

- El sitio web: Es una página que contenga todos los componentes su diseño debe ser amigable y fácil de manejar, para el cliente para que se pueda informar fácilmente sobre la empresa, sus productos con su uso y como poder adquirirlos.

Es necesario que la cooperativa Industrial de Calzado Penipe "Calzado Vinicio" para captar la atención del cliente, y posicionarse en la mente del consumidor desarrolle una fuerte imagen corporativa, que le permita diferenciarse de su competencia, a través de la aplicación del Plan de Negocios.

\section{Fundamentación legislativa}

La Cooperativa Industrial de Calzado Penipe "Calzado Vinicio", se rige a un conjunto de normas, reglamentos y leyes que son indispensables para el desarrollo de las actividades de producción y comercialización de calzado, haciendo referencia a leyes laborales, tributarias, ordenanzas municipales, medio ambiente entre ellas son:

- La Constitución del Ecuador.

- Ley de Seguridad Social.

- Ley del régimen tributaria.

- Ley de Economía Popular y Solidaria.

- Código de Trabajo.

- Código de la producción.

- Ordenanza Municipal del cantón Penipe 
MÉTODO

La metodología de la investigación fue de tipo descriptivo con un enfoque cualitativo y cuantitativo, el primero porque generó la comprensión del fenómeno social respecto a gustos y preferencias que tiene el cliente y el consumidor para interpretar y comprender la realidad social del sector en donde se va a desarrollar la comercialización del calzado, y el segundo ya que permitió recoger y analizar datos obtenidos utilizando la estadística descriptiva mediante la aplicación de modelos estadísticos que permitieron comprobar los objetivos de la investigación.

A su vez, el diseño que se usó fue cuasi experimental, dado a que no se tuvo un criterio de control y se realizó en momentos determinados, los métodos usados fueron inductivo y deductivo.

Por otro lado, la técnica e instrumentos utilizados para la recolección de datos fueron la observación; esta técnica permitió conseguir datos relativos a los posibles demandantes de la ciudad de Riobamba, además facilitó la obtención de información referente a los procesos internos de la corporación. Y la encuesta, ya que, se logró obtener información de los clientes en relaciona a sus gustos y preferencias sobre el calzado, la misma que se aplicó al mercado objetivo, y otra encuesta a las personas que laboran en la Cooperativa Industrial de Calzado.

Para la recolección de datos se aplicó dos tipos de encuestas: la primera fue a una población de 10 personas que laboran en la empresa por lo que es finito, con el objetivo de determinar el nivel de cumplimiento y fidelidad de los colaboradores de sus funciones en la gestión administrativa. Y la segunda encuesta se aplicó a los posibles consumidores en la ciudad de Riobamba. En esta oportunidad se tomó en cuenta, la población del área urbana de la ciudad de Riobamba, según proyecciones INEC para el año 2016 fue de 252.865 habitantes.

Para la investigación se trabajó con la población entre un rango de edad de 25 a 69 años, el cual fue representada por 118.982 personas, de ésta solo se usó el $25 \%$ de la muestra de 384 personas, el cual fue de 96 personas. La misma se distribuyó de la siguiente manera:

La ponderación de número de encuestas a emplearse se consideró en los siguientes rangos de edades de acuerdo a la frecuencia de gustos de las personas de la ciudad de Riobamba: de 25 - 29 (15 encuestas ) , 30-34 (20 encuestas ), 35-39 (15 encuestas ), $40-44$ ( 13 encuestas ), 45-49 ( 13 encuestas ), 50 54 (7 encuestas), 55-59 ( 5 encuestas ), 60 64 ( 4 encuestas), $65-69$ ( 4 encuestas ).

\section{RESULTADOS}

Posterior a la aplicación de las encuestas aplicadas a los clientes potenciales de la marca de calzado Vinicio en la ciudad de Riobamba, se dio a conocer los gustos y preferencias de los posibles consumidores del producto así como la información recabada a las personas que laboran en la empresa de producción industrial Calzado Penipe.

La información detallada es de la aplicación de las encuestas del antes y después de la aplicación del Plan de Negocios para fortalecer la imagen corporativa, determinar si el resultado fue positivo, también determinar si la gestión de funciones de los colaboradores tuvo mayor énfasis en sus conocimientos y aplicación en la atención al cliente.

En este sentido, se muestra los resultados obtenidos de las encuestas aplicadas a los clientes externos e internos de la empresa de Calzado Vinicio. 


\section{Clientes internos}

Conocimiento de la existencia de la misión en la Cooperativa Industrial de Calzado Penipe " Calzado Vinicio

En la tabla 1, se muestra que el $90 \%$ de las personas encuestadas en la cooperativa de Calzado Penipe "Calzado Vinicio", si tienen conocimiento de la misión de la cooperativa, mientras que el $10 \%$ no la conoce, dando como resultado que la mayoría de colaboradores si saben que existe la misión.

Tabla 1. Conocimiento de la existencia de la misión

\begin{tabular}{ccc}
\hline ALTERNATIVAS & FRECUENCIA & $\%$ \\
\hline Si & 9 & 90 \\
No & 1 & 10 \\
\hline TOTAL & $\mathbf{1 0}$ & $\mathbf{1 0 0}$
\end{tabular}

\section{Conocimiento de la misión en la Cooperativa Industrial de Calzado Penipe " Calzado} Vinicio

En la tabla 2, se muestras que el $10 \%$ de las personas encuestadas en la cooperativa de Calzado Penipe "Calzado Vinicio", sí tienen conocimiento de la misión , mientras que el 90\% no conoce cuál es el objetivo de la corporación y sus componentes para cumplirlo los mismo que detallan la misión de la empresa.

Tabla 2. Conocimiento de la Misión.

\begin{tabular}{ccc}
\hline ALTERNATIVAS & FRECUENCIA & $\%$ \\
\hline $\mathrm{Si}$ & 1 & 10 \\
No & 9 & 90 \\
\hline TOTAL & $\mathbf{1 0}$ & $\mathbf{1 0 0}$
\end{tabular}

Conocimiento de la existencia de la Visión en la Cooperativa Industrial de Calzado Penipe "Calzado Vinicio"

Se muestra en la tabla 3 que, el $90 \%$ de las personas encuestadas en la cooperativa de Calzado Penipe "Calzado Vinicio", sí tienen conocimiento, mientras que el $10 \%$ no conoce, dando como resultado que la mayoría de colaboradores si saben que existe la Visión.

Tabla 3. Conocimiento de la existencia de la Visión

\begin{tabular}{ccc}
\hline ALTERNATIVAS & FRECUENCIA & $\%$ \\
\hline $\mathrm{Si}$ & 9 & 90 \\
$\mathrm{NO}$ & 1 & 10 \\
\hline TOTAL & $\mathbf{1 0}$ & $\mathbf{1 0 0}$
\end{tabular}




\section{Conocimiento de la Visión en la Cooperativa Industrial de Calzado Penipe "Calzado Vinicio"}

El 20\% de las personas encuestadas en la cooperativa de Calzado Penipe "Calzado Vinicio", sí tienen conocimiento de la Visión, mientras que el $80 \%$ no conoce cuál es el objetivo de la corporación y sus componentes para cumplirlo los mismo que detallan la visión de la empresa.

Tabla 4. Conocimiento de la Visión

\begin{tabular}{ccc}
\hline ALTERNATIVAS & FRECUENCIA & $\%$ \\
\hline $\mathrm{Si}$ & 2 & 20 \\
No & 8 & 80 \\
\hline TOTAL & 10 & 100
\end{tabular}

\section{Conformidad con el salario mensual percibido a cambio del trabajo}

En la tabla 5, se muestra que el $100 \%$ de las personas encuestadas en la Cooperativa de Calzado Penipe "Calzado Vinicio", si están conformes con el salario que perciben mensualmente, lo cual es una fortaleza para la cooperativa al tener personal incentivado económicamente.

Tabla 5. Conformidad con el salario percibido

\begin{tabular}{ccc}
\hline ALTERNATIVAS & FRECUENCIA & $\%$ \\
\hline $\mathrm{Si}$ & 10 & 100 \\
$\mathrm{No}$ & 0 & 0 \\
\hline TOTAL & $\mathbf{1 0}$ & $\mathbf{1 0 0}$ \\
\hline
\end{tabular}

\section{Conocimiento de las funciones en el puesto de trabajo}

De esta manera se muestra que, el $60 \%$ de las personas encuestadas en la Cooperativa de Calzado Penipe "Calzado Vinicio", sí tienen conocimiento de sus funciones en cada uno de los puestos de trabajo, mientras que el $40 \%$ no conocen, de tal forma que es una debilidad para la empresa porque su personal no tiene conocimiento en su totalidad respecto a sus funciones a cumplir en cada una de las actividades.

Tabla 6. Conocimiento de las funciones en el puesto de trabajo.

\begin{tabular}{ccc}
\hline ALTERNATIVAS & FRECUENCIA & $\%$ \\
\hline $\mathrm{Si}$ & 6 & 60 \\
$\mathrm{No}$ & 4 & 40 \\
\hline TOTAL & $\mathbf{1 0}$ & $\mathbf{1 0 0}$ \\
\hline
\end{tabular}


Existencia de Manual de funciones en el cual describe claramente cuáles son las obligaciones y deberes del trabajador

Se muestra que, el $30 \%$ de las personas encuestadas en la Cooperativa de Calzado Penipe "Calzado Vinicio", manifiestan que si existe un manual de funciones, mientras que el $70 \%$ no tiene conocimiento de la existencia de dicho instrumento, motivo por el cual no tienen definido claramente cuáles son sus funciones y obligaciones.

Tabla 7. Existencia de Manual de funciones

\begin{tabular}{ccc}
\hline ALTERNATIVAS & FRECUENCIA & $\%$ \\
\hline $\mathrm{Si}$ & 3 & 30 \\
No & 7 & 70 \\
\hline TOTAL & 10 & $\mathbf{1 0 0}$ \\
\hline
\end{tabular}

Necesidad de un Manual de Funciones organizacional que describa funciones, políticas, y obligaciones a cumplir por el personal

En la tabla 8 se muestra que, el $100 \%$ de las personas encuestadas en la Cooperativa de Calzado Penipe "Calzado Vinicio", manifiestan que si es necesario diseñar este instrumento administrativo que contenga las funciones y obligaciones de cada uno de los colaboradores de la empresa para tener claramente que es lo que deben hacer en cada una de las actividades de la empresa y así tener una adecuada gestión administrativa.

Tabla 8. Necesidad de un Manual de Funciones

\begin{tabular}{ccc}
\hline ALTERNATIVAS & FRECUENCIA & $\%$ \\
\hline $\mathrm{Si}$ & 10 & 100 \\
$\mathrm{No}$ & 0 & 0 \\
\hline TOTAL & $\mathbf{1 0}$ & $\mathbf{1 0 0}$
\end{tabular}

\section{Encuestas posibles consumidores de Calzado Vinicio}

\section{Conocimiento de la marca Calzado Vinicio}

Se muestra en la tabla 9 que, el $30 \%$ de las personas encuestadas tiene conocimiento de la marca de Calzado Vinicio, en cambio un $70 \%$ no la conoce, por lo que se puede definir que no existe en los clientes un fortalecimiento de la imagen corporativa de la empresa en la ciudad de Riobamba.

Tabla 9. Conocimiento de la marca

\begin{tabular}{ccc}
\hline ALTERNATIVAS & FRECUENCIA & $\%$ \\
\hline Si & 29 & 30 \\
No & 67 & 70 \\
\hline TOTAL & 96 & 100
\end{tabular}




\section{Uso del producto de Calzado Vinicio}

En la tabla 10 se muestra que, el $16 \%$ de las personas encuestadas si ha comprado y usado, mientras que el $84 \%$ no, por lo que existe un escaso consumo del producto de Calzado Vinicio en la ciudad de Riobamba.

Tabla 10. Uso del producto

\begin{tabular}{ccc}
\hline ALTERNATIVAS & FRECUENCIA & $\%$ \\
\hline Si & 15 & 16 \\
No & 81 & 84 \\
\hline TOTAL & 96 & 100
\end{tabular}

\section{Utilización de medios de publicidad de Calzado Vinicio}

Se muestra en la tabla 11 que, el 15\% de las personas encuestadas si ha escuchado, visto y utilizado publicidad de Calzado Vinicio, mientras que el $85 \%$ no tiene conocimiento del producto a través de algún medio publicitario debido a la deficiente aplicación de marketing.

Tabla 11. Utilización de medios de publicidad

\begin{tabular}{ccc}
\hline ALTERNATIVAS & FRECUENCIA & $\%$ \\
\hline Si & 14 & 15 \\
No & 82 & 85 \\
\hline TOTAL & 96 & 100
\end{tabular}

\section{Utilización del tipo de calzado}

Se muestra que, el $49 \%$ de las personas encuestadas utiliza tipo de calzado casual, el $33 \%$ deportivo y el $18 \%$ formal, por lo que se pudo definir que existe una mayor parte de posibles consumidores que sus gustos y preferencias se dirige por calzado casual.

Tabla 12. Tipo de calzado

\begin{tabular}{ccc}
\hline ALTERNATIVAS & FRECUENCIA & \% \\
\hline Casual & 47 & 49 \\
Deportivo & 32 & 33 \\
Formal & 17 & 18 \\
\hline Total & $\mathbf{9 6}$ & $\mathbf{1 0 0}$ \\
\hline
\end{tabular}

\section{Frecuencia de consumo del producto Calzado}

Se muestra que, el $39 \%$ de las personas encuestadas compran una vez cada seis meses un par de zapatos, el $35 \%$ una vez cada tres meses, el $15 \%$ una vez al año y el $11 \%$ una vez al mes, son porcentajes muy significativos que muestran la utilización de Calzado. 
Tabla 13. Frecuencia de consumo del producto Calzado

\begin{tabular}{ccc}
\hline ALTERNATIVAS & FRECUENCIA & \% \\
\hline Una vez al mes & 11 & 11 \\
Una vez cada tres meses & 34 & 35 \\
Una vez cada seis meses & 37 & 39 \\
Una vez cada año. & 14 & 15 \\
\hline Total & $\mathbf{9 6}$ & $\mathbf{1 0 0}$ \\
\hline
\end{tabular}

\section{Lugar de adquisición del calzado}

En este sentido, se muestra que El $44 \%$ de las personas encuestadas compran el producto calzado en almacenes, el $32 \%$ en locales comerciales, y el $17 \%$ en la plaza, esto es importante para la Cooperativa de Calzado Penipe porque se puede direccionar a implantar almacenes y locales comerciales en la ciudad de Riobamba con el producto Calzado Vinicio.

Tabla 14. Lugar de adquisición del calzado

\begin{tabular}{ccc}
\hline ALTERNATIVAS & FRECUENCIA & $\%$ \\
\hline Plaza & 16 & 17 \\
Almacenes & 42 & 44 \\
Locales comerciales & 31 & 32 \\
Fábrica & 3 & 3 \\
Otros & 4 & 4 \\
\hline Total & $\mathbf{9 6}$ & $\mathbf{1 0 0}$
\end{tabular}

\section{Características del producto calzado para adquirirlo}

En la tabla 15 se muestra que, el $35 \%$ de las personas encuestadas prefieren adquirir el calzado observando el modelo, el $27 \%$ el precio, el $21 \%$ el diseño y el $17 \%$ la marca, de tal forma que se tuvo en cuenta la variedad de modelo y a un precio conveniente para los futuros clientes del producto Calzado Vinicio.

Tabla 15. Características del calzado

\begin{tabular}{ccc}
\hline ALTERNATIVAS & FRECUENCIA & \% \\
\hline Marca & 16 & 17 \\
Modelo & 34 & 35 \\
Precio & 26 & 27 \\
Diseño & 20 & 21 \\
\hline Total & $\mathbf{9 6}$ & $\mathbf{1 0 0}$ \\
\hline
\end{tabular}




\section{Disponibilidad de pago por un par de zapatos}

En la tabla 16 se muestra que el $31 \%$ de las personas encuestadas está dispuestas a pagar de $\$ 20,00$ - $\$ 30,00$, el $201 \%$ entre $\$ 10,00$ a $\$ 20,00$ el $20 \%$ de $\$ 40,00$ a 50,00 por lo que la cooperativa de Calzado Penipe "Calzado Vinicio" puede establecer el precio de venta al público considerando la disponibilidad de pago de los posibles clientes.

Tabla 16. Disponibilidad de pago por un par de zapatos

\begin{tabular}{ccc}
\hline ALTERNATIVAS & FRECUENCIA & $\%$ \\
\hline$\$ 10,00-20,00$ & 20 & 21 \\
$\$ 20,00-30,00$ & 30 & 31 \\
$\$ 30,00-40,00$ & 19 & 20 \\
$\$ 40,00-50,00$ & 8 & 8 \\
$\$ 50,00-100,00$ & 13 & 14 \\
$\$ 100,00-$ a más. & 6 & $\mathbf{6}$ \\
\hline Total & $\mathbf{9 6}$ & $\mathbf{1 0 0}$ \\
\hline
\end{tabular}

Encuestas después de la aplicación del Plan de Negocios

Conocimiento de la misión en la Cooperativa Industrial de Calzado Penipe " Calzado Vinicio"

Tabla 17. Conocimiento de la misión en la Cooperativa

\begin{tabular}{ccc}
\hline ALTERNATIVAS & FRECUENCIA & $\%$ \\
\hline $\mathrm{Si}$ & 8 & 80 \\
No & 2 & 20 \\
\hline TOTAL & 10 & 100
\end{tabular}

Se muestra que, el $80 \%$ de las personas encuestadas en la Cooperativa de Calzado Penipe "Calzado Vinicio", si tienen conocimiento, mientras que el $20 \%$ no conoce, dando como resultado que la mayoría de colaboradores si conocen la Misión.

\section{Conocimiento de la Visión en la Cooperativa Industrial de Calzado Penipe " Calzado Vinicio"}

Se muestra en la tabla 18 , que, el $70 \%$ de las personas encuestadas en la cooperativa de Calzado Penipe "Calzado Vinicio", si tienen conocimiento, mientras que el 30\% no conoce, dando como resultado que la mayoría de colaboradores si conocen la Visión. 
Tabla 18. Conocimiento de la Visión

\begin{tabular}{ccc}
\hline ALTERNATIVAS & FRECUENCIA & $\%$ \\
\hline $\mathrm{Si}$ & 7 & 70 \\
$\mathrm{NO}$ & 3 & 30 \\
\hline TOTAL & $\mathbf{1 0}$ & $\mathbf{1 0 0}$
\end{tabular}

\section{Conocimiento de las funciones en el puesto de trabajo}

Es notable, que el $100 \%$ de las personas encuestadas en la cooperativa de Calzado Penipe "Calzado Vinicio", si tienen conocimiento, de las funciones en el puesto de trabajo, luego de aplicar el plan organizacional.

Tabla 19. Conocimiento de las funciones en el puesto de trabajo

\begin{tabular}{ccc}
\hline ALTERNATIVAS & FRECUENCIA & $\%$ \\
\hline $\mathrm{Si}$ & 10 & 100 \\
$\mathrm{NO}$ & 0 & 0 \\
\hline TOTAL & $\mathbf{1 0}$ & $\mathbf{1 0 0}$
\end{tabular}

Existencia de Manual de Funciones el cual describe claramente cuáles son sus obligaciones y deberes

Tabla 20. Existencia del manual de funciones

\begin{tabular}{ccc}
\hline ALTERNATIVAS & FRECUENCIA & $\%$ \\
\hline $\mathrm{Si}$ & 8 & 80 \\
$\mathrm{No}$ & 2 & 20 \\
\hline
\end{tabular}

Se evidencia que, el $80 \%$ de las personas encuestadas en la cooperativa de Calzado Penipe "Calzado Vinicio", si tienen conocimiento de la existencia del Manual de Funciones, mientras que el $20 \%$ no conoce, luego de aplicar el plan organizacional.

\section{Encuestas de los posibles clientes y consumidores}

\section{Conocimiento de la marca}

Se muestra que, el $58 \%$ de las personas encuestadas tiene conocimiento sobre la marca de Calzado Vinicio, mientras que el $42 \%$ no tiene conocimiento, en relación a los resultados antes de la aplicación del Plan de negocios se ha incrementado en un $28 \%$ durante el período de 2 meses, por lo que es un indicador aceptable y con tendencias de seguir incrementado la marca en la ciudad de Riobamba. 
Tabla 21. Conocimiento de la marca

\begin{tabular}{ccc}
\hline ALTERNATIVAS & FRECUENCIA & $\%$ \\
\hline $\mathrm{Si}$ & 56 & 58 \\
$\mathrm{NO}$ & 40 & 42 \\
\hline TOTAL & $\mathbf{1 0}$ & $\mathbf{1 0 0}$
\end{tabular}

\section{Uso del producto de Calzado Vinicio}

Se muestra que, el 33\% de las personas encuestadas han consumido Calzado Vinicio, mientras que el $67 \%$ no tiene conocimiento, en relación a los resultados antes de la aplicación del Plan de negocios se ha incrementado en un 18\% durante el periodo de 2 meses, por lo que demostró que han elevado las ventas, dando validez a la aplicación de la publicidad de la empresa.

Tabla 22. Consumo del producto Calzado Vinicio

\begin{tabular}{ccc}
\hline ALTERNATIVAS & FRECUENCIA & \% \\
\hline $\mathrm{Si}$ & 23 & 33 \\
$\mathrm{No}$ & 46 & 67 \\
\hline TOTAL & $\mathbf{9 6}$ & $\mathbf{1 0 0}$
\end{tabular}

\section{Utilización de medios de publicidad}

Se muestra que, el 31\% de las personas encuestadas si han escuchado, visto o recibido algún tipo de publicidad de Calzado Vinicio, lo que significa que se incrementó en un $17 \%$ mayor al que se obtuvo antes de la aplicación del Plan de negocios, por lo que dio éxito en la aplicación de la campaña publicitaria.

Tabla 23. Utilización de medios de publicidad

\begin{tabular}{ccc}
\hline ALTERNATIVAS & FRECUENCIA & \% \\
\hline $\mathrm{Si}$ & 30 & 31 \\
$\mathrm{No}$ & 66 & 69 \\
\hline TOTAL & $\mathbf{1 0}$ & $\mathbf{1 0 0}$
\end{tabular}

\section{CONCLUSIONES}

Posterior al desarrollo de la investigación, y aplicación de los instrumentos para la recolección de datos, se logró con el objetivo principal, ya que se diseñó y aplicó el plan organizacional, lo que permitió que los colaboradores de la empresa obtuvieran el conocimiento total sobre la misión y visión, las funciones y deberes que deben cumplir en cada una de las actividades para la producción y comercialización de calzado.

Adicionalmente se fortaleció la imagen de la Cooperativa Industrial de Calzado Vinicio en la ciudad de Riobamba, por el diseño del plan de marketing que se centró en la aplicación de medios publicitarios: página web, facebook, google plus, volantes, dípticos con lo que se alcanzó incrementar en un $21 \%$ 
el conocimiento de la imagen corporativa en los clientes y futuros consumidores del producto en la ciudad de Riobamba.

Por último, con la aplicación del Plan de Financiero se incrementó en un $25 \%$ el consumo del producto de esta forma elevando en nivel de ventas y por ende la rentabilidad de la empresa.

\section{REFERENCIAS}

Almoguera, S. (2009). Plan de Negocios. Madrid, España: ESINE Centro de Estudios Técnicos empresariales

Corporación Financiera Nacional. (2013)

Duarte, C. (2012). La imagen corporativa. Gerencie.com

http://www.gerencie.com/laimagencorporativa.html

Lamb, B.; Hair, J., y McDaniel, C. (2011). Marketing. 11e. Mason OH, USA: Cengage Learning

Martínez. (2013). Plan de Negocios
Monroy, E. (2014). Análisis del proceso administrativo desde varios autores.

Recuperado el 10 de Junio de 2016, de Análisis del proceso administrativo desde varios autores

Pujol, B. (2009). Diccionario de Marketing. Madrid - España: Cultural S.A.

Ruiz. (2011). Plan de Negocios en Pymes

Sapag, Ch. (2007). Proyectos de inversiones: Formulación y Evaluación. Editorial Pearson Educación. México

Vanella. (2012). Guía del emprendedor: Claves para organizar un negocio exitoso. Buenos Aires: Electrónica

Villarán, K. (2009). Plan de Negocios. Lima: Nathan Associates Inc

Walker, O.; Boyd, M.; Mullins, J., y Larréché, J. (2005). Marketing estratégico: Enfoque de toma de decisiones. (4a. ed.). México: McGraw-Hill 